\begin{tabular}{|c|l|}
\hline Title & RNF43 interacts with NEDL1 and regul ates p53-mediated transcription \\
\hline Author(s) & $\begin{array}{l}\text { Shinada, Keisuke; Tsukiyama, Tadasuke; Sho, Takuya; Okumura, Fumi hiko; A saka, Masahiro; Hatakey ama, } \\
\text { Shigetsugu }\end{array}$ \\
\hline Citation & $\begin{array}{l}\text { Biochemical and Biophysical Research Communications, 404(1), 143-147 } \\
\text { https://doi.org/10.1016/.bbrc.2010.11.082 }\end{array}$ \\
\hline Issue Date & 2011-01-07 \\
\hline Doc URL & http://hdl.handle.net/2115/44929 \\
\hline Type & article (author version) \\
\hline File Information & BBRC4041_143-147.pdf \\
\hline
\end{tabular}

Instructions for use 


\title{
RNF43 interacts with NEDL1 and regulates p53-mediated transcription
}

\author{
Keisuke Shinada $^{\text {a,b }}$, Tadasuke Tsukiyama ${ }^{\text {a }}$, Takuya Sho ${ }^{\text {a,b }}$, Fumihiko Okumura ${ }^{\text {, }}$ \\ Masahiro Asaka ${ }^{\text {b }}$ and Shigetsugu Hatakeyama ${ }^{\text {a, } *}$
}

${ }^{a}$ Department of Biochemistry, Hokkaido University Graduate School of Medicine, Sapporo, Hokkaido 060-8638, Japan

${ }^{\mathrm{b}}$ Department of Gastroenterology and Hematology, Hokkaido University Graduate School of Medicine, Sapporo 060-8638, Japan

* Corresponding author: Shigetsugu Hatakeyama, Department of Biochemistry, Hokkaido University Graduate School of Medicine, Kita15, Nishi7, Kita-ku, Sapporo, Hokkaido 060-8638, Japan. Tel.: +81 11706 5899; fax: +81 117065169.

E-mail address: hatas@med.hokudai.ac.jp (S. Hatakeyama)

Key words: RNF43; p53; NEDL1; ubiquitin; colorectal cancer 


\begin{abstract}
The ubiquitin-proteasomal system plays a crucial role in oncogenesis in colorectal tissues. Recent studies have shown that stability of $\beta$-catenin, which functions as an oncogene for colorectal cancer, is regulated by ubiquitin-mediated degradation. It has been reported that a putative E3 ubiquitin ligase, RNF43, is highly expressed in human colorectal carcinoma and that RNF43 promotes cell growth. However, the involvement of RNF43 in carcinogenesis has not been fully elucidated. In this study, we found by using yeast two-hybrid screening that RNF43 binds to NEDD-4-like ubiquitin-protein ligase-1 (NEDL1), which enhances pro-apoptotic activity by p53. In addition, we found that RNF43 also interacts with p53 and that RNF43 suppresses transcriptional activity of p53 in H1299 cells and attenuates apoptosis induced by ultraviolet irradiation. These findings suggest that RNF43 is associated with p53-mediated apoptosis in collaboration with NEDL1 in colorectal carcinogenesis.
\end{abstract}




\section{Introduction}

Colorectal cancer (CRC) is one of the leading causes of cancer death in the Western world in both males and females [1,2]. Approximately 50\% of patients eventually develop metastatic disease, for which palliative systemic treatment is usually administered. The treatment options for patients with metastatic colorectal cancer (mCRC) have changed considerably in recent years. Although a combination of 5-fluorouracil and leucovorin has so far been the only effective treatment for mCRC, irinotecan, oxaliplatin, anti-VEGF antibody (bevacizumab) and anti-EGFR antibodies (cetuximab and panitumumab) have been used as therapeutic drugs against mCRC. However, even though these treatments have been established, mCRC remains a lethal disease.

It has been reported that $\mathrm{p} 53, \mathrm{NF}-\kappa \mathrm{B}, \mathrm{Bcl}-2, \mathrm{APC} / \beta$-catenin and cyclooxygenase 2 (COX-2) are associated with colorectal tumorigenesis by affecting colonic epithelial cell apoptosis [3-5]. Since the cytotoxic action of most chemotherapeutic drugs is often mediated by the activation of apoptotic pathways, altered expression of genes encoding apoptotic proteins may produce drug resistance [6]. p53 as the guardian of the genome plays a central role in DNA damage response. p53 is the most frequently mutated gene in human cancers, and two mechanisms that control tumor growth are disrupted in carcinomas: cell cycle arrest and apoptosis. In response to DNA damage, p53 is regulated and stabilized via a series of posttranslational modifications including phosphorylation, acetylation and ubiquitination.

Ubiquitination is a versatile posttranslational modification mechanism used by eukaryotic cells mainly to control protein levels through proteasome-mediated 
proteolysis. Ubiquitin conjugation is achieved by several enzymes that act in concert, including a ubiquitin-activating enzyme (E1), a ubiquitin-conjugating enzyme (E2), and a ubiquitin-protein ligase (E3) $[7,8]$. E3 is thought to be the component of the ubiquitin conjugation system that is most directly responsible for substrate recognition. Enzymes belonging to class E3 that have been identified include members of the RING, HECT (homologous to E6-AP carboxyl terminus), and U-box families of proteins [9-11]. The RING finger domain was first identified in the human protein RNG1 in the early 1990`s. The RING finger domain contains eight metal-binding residues that coordinate two zinc atoms in an interleaved pattern for folding and biological actions [12]. RING finger proteins act by mediating diverse protein-protein interactions.

Ring-finger protein 43 (RNF43), which has been identified as a RING-type E3 ubiquitin ligase, is highly expressed in colorectal cancers. Overexpression of RNF43 resulted in a significant enhancement of cell growth and knockdown of its expression by siRNAs exerted a growth-suppressing effect [13]. These findings suggest that RNF43 plays a role in colorectal carcinogenesis. RNF43 was also identified as a tumor-associated antigen of colorectal cancer (CRC). A tumor-associated antigen contains epitope peptides that promote antitumor immune response for cancer immunotherapy [14]. However, the role of RNF43 in the progression of carcinogenesis has not been fully elucidated.

A novel HECT-type E3 ubiquitin ligase, NEDL1, has been reported to be expressed in neuronal tissues including the spinal cord and to selectively bind to and ubiquitinate mutant (but not wild-type) SOD1 and Dvl1 [15]. Furthermore, it has been reported that NEDL1 interacts with p53 and enhances transcriptional activity of p53 and p53-mediated apoptotic cell death. These findings suggest that NEDL1 regulates cell 
proliferation and differentiation, stress responses, and DNA-damage responses $[15,16]$.

In this study, we found that RNF43 interacts with NEDL1 and that overexpression of RNF43 suppresses the transcriptional activity of p53 and UV-induced apoptosis. It is likely that RNF43 is associated with p53-associated apoptotic cell death via interaction with NEDL1 in colorectal carcinogenesis. 


\section{Materials and methods}

Cell culture. HEK293T and HCT116 cell lines were cultured under an atomosphere of $5 \% \mathrm{CO}_{2}$ at $37^{\circ} \mathrm{C}$ in DMEM (Sigma) supplemented with $10 \%$ fetal bovine serum (Invitrogen). H1299 cell lines were grown in RPMI1640 (Sigma) medium supplemented with $10 \%$ fetal bovine serum (Invitrogen).

Plasmid construction. Human RNF43 was amplified by RT-PCR from the colon cancer cell line SW480 with 5'-AGCATGAGTGGTGGCCACCAG-3' (RNF43-sense) and 5'-ATCTCACACAGCCTGTTCAC-3' (RNF43-antisense). The amplified fragment was subcloned into pBluescript II KS ${ }^{+}$(Stratagene). RNF43 cDNAs digested with Hind III/Xho I were cloned into pFLAG (Sigma) at the carboxy-terminus or into pcDNA3 (Invitrogen) with HA at the carboxy-terminus. pF1KA0322 containing NEDL1 cDNA was obtained from Kazusa DNA Research Institute and then subcloned into pCI (Promega). An expression vector containing p53 cDNA was subcloned from pcDNA1-p53[17] into p5xFLAG .

Yeast two-hybrid screening. Yeast two-hybrid screening was performed with a HeLa cDNA library (Clonetech) as described previously [18]. Complementary DNA encoding 515-784 amino acid residues of human RNF43 was used as a bait.

Antibodies and reagents. The antibodies used were as follows: mouse monoclonal anti-FLAG (M2, Sigma), mouse monoclonal anti-HA (HA.11/16B12, Covance), rabbit polyclonal anti-hNEDL1 (Sigma), mouse monoclonal anti- $\beta$-actin (AC15, Sigma), and mouse monoclonal anti-p53 (Santa Cruz).

Transfection, immunoprecipitation, and immunoblot analysis. HEK293T cells were transfected by the calcium phosphate method. Thirty-six h after transfection, the cells 
were lysed in a solution containing $50 \mathrm{mM}$ Tris- $\mathrm{HCl}(\mathrm{pH} 7.4), 150 \mathrm{mM} \mathrm{NaCl}, 1 \%$ Triton-X 100, leupeptin $(10 \mu \mathrm{g} / \mathrm{ml}), 1 \mathrm{mM}$ phenylmethylsulfonyl fluoride, $400 \mu \mathrm{M}$ $\mathrm{Na}_{3} \mathrm{VO}_{4}, 400 \mu \mathrm{M}$ EDTA, $10 \mathrm{mM} \mathrm{NaF}$, and $10 \mathrm{mM}$ sodium pyrophosphate. The cell lysates were centrifuged at $16,000 \mathrm{~g}$ for $20 \mathrm{~min}$ at $4^{\circ} \mathrm{C}$ and the resulting supernatant was incubated with antibodies for $2 \mathrm{~h}$ at $4^{\circ} \mathrm{C}$. Protein A-Sepharose (Amersham Biosciences) that had been equilibrated with the same solution was added to the mixture, which was then rotated for $1 \mathrm{~h}$ at $4^{\circ} \mathrm{C}$. The resin was separated by centrifugation, washed five times with ice-cold lysis buffer, and then boiled in SDS sample buffer. Immunoblot analysis was performed with primary antibodies, horseradish peroxidase-conjugated antibodies to mouse or rabbit immunoglobulin G (1:20,000 dilution, Promega), and an enhanced chemiluminescence system (ECL, Amersham Pharmacia).

Dual-luciferase assay. p53-responsive element was digested from pp53-Box RE-TK-hRluc (F) (RIKEN) and subcloned into pGL4.20 (Promega) (pGL4.20-pp53). H1299 cells were seeded in 24-well plates at $4 \times 10^{4}$ cells per well and incubated at $37^{\circ} \mathrm{C}$ with $5 \% \mathrm{CO}_{2}$ for $24 \mathrm{~h}$. Cells were then co-transfected with p53 expression vector (100 ng), pGL4.20-pp53 (100 ng) and pRL-TK (10 ng) with or without RNF43 expression vector (50 and $250 \mathrm{ng}$ ) using Lipofectamine LTX (Invitrogen). The cells were incubated for $38 \mathrm{~h}$ and then assayed for luciferase activity with a Dual-Luciferase Reporter Assay System (Promega).

Establishment of stable transfectants. Retroviral expression vectors containing FLAG-tagged RNF43 cDNA were constructed with pMX-puro. For retrovirus-mediated gene expression, HCT116 cells were infected with retroviruses produced by Plat-A packing cells. Cells were then cultured in the presence of puromycin $(0.5 \mu \mathrm{g} / \mathrm{mL})$ for 1 week. HCT116 cell lines were prepared by transfection with 
pCAGI-puro-RNF43-FLAG or pCAGI-puro [19] by electroporation $(250 \mathrm{~V}, 960 \mu \mathrm{F})$. Twenty-four h after transfection, puromycin $(0.5 \mu \mathrm{g} / \mathrm{mL})$ was added to the cultures and single colonies were selected. Expression of RNF43 was confirmed by immunoblot analysis.

Apoptosis assay. Assessment of apoptosis was performed by measurement of the sub- $\mathrm{G}_{1}$ fraction and detection of cleaved caspase-3. For cleaved caspase-3, HCT116 cells were incubated with or without cDDP $(50 \mu \mathrm{mol} / \mathrm{L})$ for $14 \mathrm{~h}$. Cell lysates were then subjected to immunoblot analysis with anti-caspase-3 (1:1,000 dilution; 3G2, Cell Signaling) and anti-cleaved caspase-3 (Asp ${ }^{175} ; 1,000$ dilution; 5A1, Cell Signaling) antibodies. For sub-G $\mathrm{G}_{1}$ peak, HCT116 cells were exposed to UV $\left(40 \mathrm{~J} / \mathrm{cm}^{2}\right)$ for various times. The cells were treated with propidium iodide and then the sub- $\mathrm{G}_{1}$ fraction was analyzed by a FACSCalibur flow cytometer (Becton Dickingson). 


\section{Results}

RNF43 interacts with NEDL1

It has been reported that RNF43 is highly expressed in colorectal cancers and that overexpression of RNF43 causes a significant enhancement of cell growth [13]. It is important to identify the proteins interacting with RNF43 for elucidating the molecular mechanism of cell proliferation by RNF43. To identify proteins that interact with RNF43, we screened the pACT2 HeLa cDNA library by using pBTM116-hRNF43 plasmid as a bait. Sixty-six positive clones were isolated on Leu-Trp-His-deficient medium from $1.2 \times 10^{5}$ transformants. One of the positive clones had sequence identities with NEDL1 (NEDD-4-like ubiquitin-protein ligase-1). We confirmed that RNF43 interacts with NEDL1 by using a $\beta$-galactosidase assay (Fig. 1A). CHIP and EKN1 cDNAs were used for positive controls [20]. Next, we investigated whether RNF43 physically interacts with NEDL1 in mammalian cells. We transfected expression vectors encoding FLAG-tagged RNF43 and/or NEDL1 into HEK293T cells. Cell lysates were subjected to immunoprecipitation with anti-FLAG antibody and the resulting precipitates were subjected to immunoblot analysis with anti-FLAG or NEDL1 antibody. An in vivo binding assay showed that RNF43 interacts with full-length (FL) NEDL1 as well as truncated NEDL1 (326-1466 amino acids), which contains an RNF43-interacting region identified by yeast-two hybrid screening (Fig. 1B).

Interaction of RNF43 with p53 
It has been reported that NEDL1 interacts with p53 and enhances p53-mediated apoptotic cell death [16]. We examined whether RNF43 also interacts with p53. We transfected expression vectors encoding FLAG-tagged p53 and/or HA-tagged RNF43 into HEK293T cells. Cell lysates were subjected to immunoprecipitation with an anti-FLAG antibody and the resulting precipitates were subjected to immunoblot analysis with anti-HA antibody. Immunoblot analysis showed that FLAG-tagged p53 was coimmunoprecipitated with HA-tagged RNF43 in HEK293T cells (Fig. 2A). Next, we verified the interaction between FLAG-tagged RNF43 and endogenous p53 (Fig. 2B). Taken together, these findings suggest that RNF43 interacts with p53 and NEDL1.

RNF43 suppresses p53 mediated transcriptional activity

Since it has been reported that NEDL1 enhances transcriptional activity of p53[16], we examined the effect of RNF43 on p53-mediated transcriptional activity. A luciferase reporter construct containing p53-responsive element were transfected with several amounts of RNF43 and/or p53 expression vectors into H1299 cells and the luciferase activity was measured. Luciferase assays showed that RNF43 suppressed p53-mediated transactivation in a dose-dependent manner (Fig. 3).

RNF43 suppresses $U V$-induced apoptosis

Since it has been reported that overexpression of RNF43 causes cell proliferation, resulting in enhancement of oncogenic activity, we hypothesized that RNF43 regulates 
p53-mediated apoptosis. To confirm the effect of RNF43 on caspase activation, immunoblot analysis was performed to analyze the cleaved form of caspase- 3 after cDDP treatment. Immunoblot analysis showed that overexpression of RNF43 attenuates the cleavage form of caspase-3 compared with the control (Fig. 4A). Moreover, to examine the effect of RNF43 on p53-dependent apoptosis, we checked the sub- $\mathrm{G}_{1}$ fraction after UV irradiation by flowcytometric analysis. Flowcytometric analysis showed that RNF43 decreased the sub- $\mathrm{G}_{1}$ fraction as an apoptotic fraction compared with the control, suggesting that overexpression of RNF43 suppresses UV-induced apoptosis (Fig. 4B). These results suggest that RNF43 suppresses apoptosis that is mediated by $\mathrm{p} 53$. 


\section{Discussion}

In this study, we showed that RNF43, which is highly expressed in colorectal cancers, interacts with NEDL1, which binds to and regulates p53, and that RNF43 also interacts with p53 and suppresses p53-mediated transcription. p53 is one of the important tumor suppressors and integrates multiple stress signals into an anti-tumor response. p53 protein normally induces G1 cell-cycle arrest to facilitate DNA repair during replication or it induces apoptosis. We showed that RNF43 suppresses apoptosis induced by UV irradiation or cDDP treatment. These findings suggest that RNF43 regulates cell growth and apoptosis via NEDL1 and p53.

It has been reported that several pathways are involved in colorectal carcinogenesis [21]. The carcinogenic pathway in colorectal cancers is characterized by allelic losses on chromosome 5q (APC), 7p (p53), and 18q (DCC/SMAD4) and is therefore called the chromosomal instability (CNI) pathway. Patients with CRC involving a p53 mutation have worse survival than do patients whose cancers do not have a p53 mutation [22]. Thus, P53-mediated apoptosis has an important role in colorectal carcinogenesis. Although p53 mutations are generally considered to occur at the stage of transformation from adenoma to adenocarcinoma in this pathway, it has been reported that p53 abnormalities, either mutation or loss of heterozygosity, exist in $4-26 \%$ of adenomas, $50 \%$ of adenomas with invasive foci, and $50-75 \%$ of CRCs having impaired function of p53 [23]. RNF43 is overexpressed in colon adenoma as well as colorectal cancers [24]. p53 mutation with adenoma suggests that p53 with adenoma might contribute to the proapoptotic function of $\mathrm{p} 53$. It has been reported that NEDL1 enhances the transcriptional activity of p53 and p53-mediated apoptotic cell death. We showed that 
RNF43 interacts with NEDL1 and suppresses p53-mediated transcriptional activity, suggesting that RNF43 functions as a negative regulator of NEDL1. Taken together, RNF43 likely plays a crucial role for regulating wild-type p53-associated apoptotic cell death. However, the detailed molecular mechanism of RNF43 remains to be unknown.

It has been reported that p53 is regulated by posttranslational modifications including ubiquitination, phosphorylation and methylation and that posttranslational modifications of p53 are associated with its functions, including DNA repair, cell cycle arrest and apoptosis [25]. p53 is ubiquitinated by Mdm2 E3 ligase and acetylation of p53 destabilizes the p53-Mdm2 interaction, suggesting that acetylation of p53 is indispensable for p53 activation [26]. Acetylation levels of p53 can be regulated via deacetylation by an HDAC1 (histone deacetylase 1)-containing complex or by NAD-dependent histone deacetylase $\operatorname{Sir} 2 \alpha$ (silent information regulator $2 \alpha$ ). These findings suggest that deacetylation represses p53-dependent transcriptional activation, cell-cycle arrest and apoptosis [27]. RNF43 and NEDL1 likely have ubiquitin ligase activities because RNF43 and NEDL1 have a RING domain and a HECT domain, respectively. Overexpression of NEDL1 enhances p53-mediated transcription, suggesting that NEDL1 does not degrade p53 via the ubiquitin-proteasomal pathway [16]. On the other hand, we showed that RNF43 interacts with NEDL1 and inhibits p53-mediated transcription, suggesting that RNF43 is an E3 ubiquitin ligase for NEDL1 or $\mathrm{p} 53$.

In conclusion, we showed that RNF43 suppresses transcriptional activity of p53 and apoptosis induced by UV or cDDP treatment. However, it is unclear whether interaction of RNF43 with NEDL1 and p53 contributes to posttranslational modifications of p53. Further biochemical studies and genetic approaches using transgenic or knock-out mice 
will be necessary to clarify the relationship between RNF43 and carcinogenesis in the colorectal region. 


\section{Acknowledgments}

We thank T. Kitamura, Kazusa DNA Research Institute and RIKEN BioResource Center for the plasmids and Y. Soida for help in preparing the manuscript.

This work was supported in part by a research grant from Grant-in-Aid for Scientific Research on Priority Areas from the Ministry of Education, Culture, Sports, Science and Technology, and Ono Cancer Research Fund (to S. Hatakeyama). 


\section{References}

[1] J. Ferlay, P. Autier, M. Boniol, M. Heanue, M. Colombet, P. Boyle, Estimates of the cancer incidence and mortality in Europe in 2006, Ann. Oncol. 18 (2007) $581-592$.

[2] A. Jemal, R. Siegel, J. Xu, E. Ward, Cancer statistics, 2010, CA Cancer. J. Clin. 60 (2010) 277-300.

[3] T. Reya, H. Clevers, Wnt signalling in stem cells and cancer, Nature 434 (2005) $843-850$

[4] J.C. Reed, Dysregulation of apoptosis in cancer, J. Clin. Oncol. 17 (1999) 2941-2953.

[5] A. Greenhough, H.J. Smartt, A.E. Moore, H.R. Roberts, A.C. Williams, C. Paraskeva, A. Kaidi, The COX-2/PGE2 pathway: key roles in the hallmarks of cancer and adaptation to the tumour microenvironment, Carcinogenesis 30 (2009) 377-386.

[6] R.W. Johnstone, A.A. Ruefli, S.W. Lowe, Apoptosis: a link between cancer genetics and chemotherapy, Cell 108 (2002) 153-164.

[7] A. Hershko, A. Ciechanover, The ubiquitin system, Annu. Rev. Biochem. 67 (1998) 425-479.

[8] C.M. Pickart, Mechanisms underlying ubiquitination, Annu. Rev. Biochem. 70 (2001) 503-533.

[9] J.M. Huibregtse, M. Scheffner, S. Beaudenon, P.M. Howley, A family of proteins structurally and functionally related to the E6-AP ubiquitin-protein ligase, Proc. Natl. Acad. Sci. USA 92 (1995) 5249.

[10] K.L. Lorick, J.P. Jensen, S. Fang, A.M. Ong, S. Hatakeyama, A.M. Weissman, 
RING fingers mediate ubiquitin-conjugating enzyme (E2)-dependent ubiquitination, Proc. Natl. Acad. Sci. USA 96 (1999) 11364-11369.

[11] S. Hatakeyama, M. Yada, M. Matsumoto, N. Ishida, K.I. Nakayama, U box proteins as a new family of ubiquitin-protein ligases, J. Biol. Chem. 276 (2001) 33111-33120.

[12] A.D. Capili, E.L. Edghill, K. Wu, K.L. Borden, Structure of the C-terminal RING finger from a RING-IBR-RING/TRIAD motif reveals a novel zinc-binding domain distinct from a RING, J. Mol. Biol. 340 (2004) 1117-1129.

[13] R. Yagyu, Y. Furukawa, Y.M. Lin, T. Shimokawa, T. Yamamura, Y. Nakamura, A novel oncoprotein RNF43 functions in an autocrine manner in colorectal cancer, Int. J. Oncol. 25 (2004) 1343-1348.

[14] N. Uchida, T. Tsunoda, S. Wada, Y. Furukawa, Y. Nakamura, H. Tahara, Ring finger protein 43 as a new target for cancer immunotherapy, Clin. Cancer Res. 10 (2004) 8577-8586.

[15] K. Miyazaki, T. Fujita, T. Ozaki, C. Kato, Y. Kurose, M. Sakamoto, S. Kato, T. Goto, Y. Itoyama, M. Aoki, A. Nakagawara, NEDL1, a novel ubiquitin-protein isopeptide ligase for dishevelled-1, targets mutant superoxide dismutase-1, J. Biol. Chem. 279 (2004) 11327-11335.

[16] Y. Li, T. Ozaki, H. Kikuchi, H. Yamamoto, M. Ohira, A. Nakagawara, A novel HECT-type E3 ubiquitin protein ligase NEDL1 enhances the p53-mediated apoptotic cell death in its catalytic activity-independent manner, Oncogene 27 (2008) 3700-3709.

[17] A. Goga, X. Liu, T.M. Hambuch, K. Senechal, E. Major, A.J. Berk, O.N. Witte, C.L. Sawyers, p53 dependent growth suppression by the c-Abl nuclear tyrosine 
kinase, Oncogene 11 (1995) 791-799.

[18] F. Okumura, S. Hatakeyama, M. Matsumoto, T. Kamura, K.I. Nakayama, Functional regulation of FEZ1 by the U-box-type ubiquitin ligase E4B contributes to neuritogenesis, J. Biol. Chem. 279 (2004) 53533-53543.

[19] H. Niwa, K. Yamamura, J. Miyazaki, Efficient selection for high-expression transfectants with a novel eukaryotic vector, Gene 108 (1991) 193-199.

[20] S. Hatakeyama, M. Matsumoto, M. Yada, K.I. Nakayama, Interaction of U-box-type ubiquitin-protein ligases (E3s) with molecular chaperones, Genes Cells 9 (2004) 533-548.

[21] W.M. Grady, Genomic instability and colon cancer, Cancer Metastasis Rev. 23 (2004) 11-27.

[22] A. Russo, V. Bazan, B. Iacopetta, D. Kerr, T. Soussi, N. Gebbia, The TP53 colorectal cancer international collaborative study on the prognostic and predictive significance of p53 mutation: influence of tumor site, type of mutation, and adjuvant treatment, J. Clin. Oncol. 23 (2005) 7518-7528.

[23] A. Leslie, F.A. Carey, N.R. Pratt, R.J. Steele, The colorectal adenoma-carcinoma sequence, Br. J. Surg. 89 (2002) 845-860.

[24] T. Sugiura, A. Yamaguchi, K. Miyamoto, A cancer-associated RING finger protein, RNF43, is a ubiquitin ligase that interacts with a nuclear protein, HAP95, Exp. Cell Res. 314 (2008) 1519-1528.

[25] Y. Tang, W. Zhao, Y. Chen, Y. Zhao, W. Gu, Acetylation is indispensable for p53 activation, Cell 133 (2008) 612-626.

[26] K.H. Vousden, C. Prives, Blinded by the Light: The Growing Complexity of p53, Cell 137 (2009) 413-431. 
[27] J. Luo, F. Su, D. Chen, A. Shiloh, W. Gu, Deacetylation of p53 modulates its effect on cell growth and apoptosis, Nature 408 (2000) 377-381. 


\section{Figure legends}

Fig. 1. RNF43 interacts with NEDL1. (A) Yeast two-hybrid screening for identifying RNF43-interacting proteins. Human NEDL1 was identified as an RNF43-interacting protein using a HeLa cDNA library. Mock indicates an empty vector (pBTM116) as a negative control. CHIP and EKN1 cDNAs were used for positive controls. (B) In vivo binding assay of RNF43 with NEDL1. HEK293T cells were transfected with expression plasmids encoding FLAG-tagged RNF43 and full-length NEDL1 or truncated NEDL1 mutant (326-1466). Whole cell lysates (WCL) were immunoprecipitated with anti-FLAG antibody and immunoblotted with anti-FLAG or anti-NEDL1 antibody.

Fig. 2. Interaction between RNF43 and p53. (A) In vivo binding assay of RNF43 with p53. HEK293T cells were transfected with expression plasmids encoding HA-tagged RNF43 and FLAG-tagged p53. Thirty-six $h$ after transfection, WCL were immunoprecipitated with anti-FLAG antibody and immunoblotted with anti-FLAG or anti-HA antibody. (B) Interaction of RNF43 with endogenous p53. HEK293T cells were transfected with or without FLAG-tagged RNF43. Thirty-six h after transfection, WCL were immunoprecipitated with anti-FLAG antibody and immunoblotted with anti-FLAG or anti-p53 antibody.

Fig. 3. RNF43 suppresses transcriptional activity of p53. H1299 cells were co-transfected with an expression plasmid encoding p53 (50 ng), luciferase reporter plasmid containing p53-responsive element (100 ng) and Renilla luciferase plasmid together (10 ng) with or without several amounts of expression plasmid for RNF43 (0, 
50 or $250 \mathrm{ng}$ ). Total amount of plasmid DNA per transfection (500 ng) was adjusted with pcDNA3. Thirty-six $\mathrm{h}$ after transfection, cell lysates were prepared and their luciferase activity was measured. Data were normalized to Renilla luciferase activity. Data are means \pm SD of values from three independent experiments. $P$ values for indicated comparisons were determined by Student's $t$ test.

Fig. 4. RNF43 suppresses apoptosis. (A) RNF43 inhibits cleavage of caspase-3 by cDDP treatment. HCT116 cell lines stably expressing FLAG-tagged RNF43, which was transfected by electroporation, were incubated with cDDP $(50 \mu \mathrm{mol} / \mathrm{L})$ for indicated times. Cell lysates were then subjected to immunoblot analysis with an antibody to cleaved caspase-3. (B) RNF43 attenuates DNA fragmentation by UV irradiation. HCT116 cell lines stably expressing FLAG-tagged RNF43, which was infected by a retroviral expression system, were treated with UV irradiation $\left(40 \mathrm{~J} / \mathrm{cm}^{2}\right)$. At the indicated time periods, cells were analyzed by a flow cytometer and the percentages of sub-G1 fraction were measured 
A

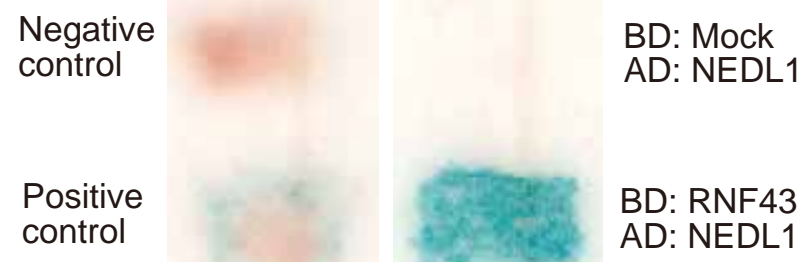

B

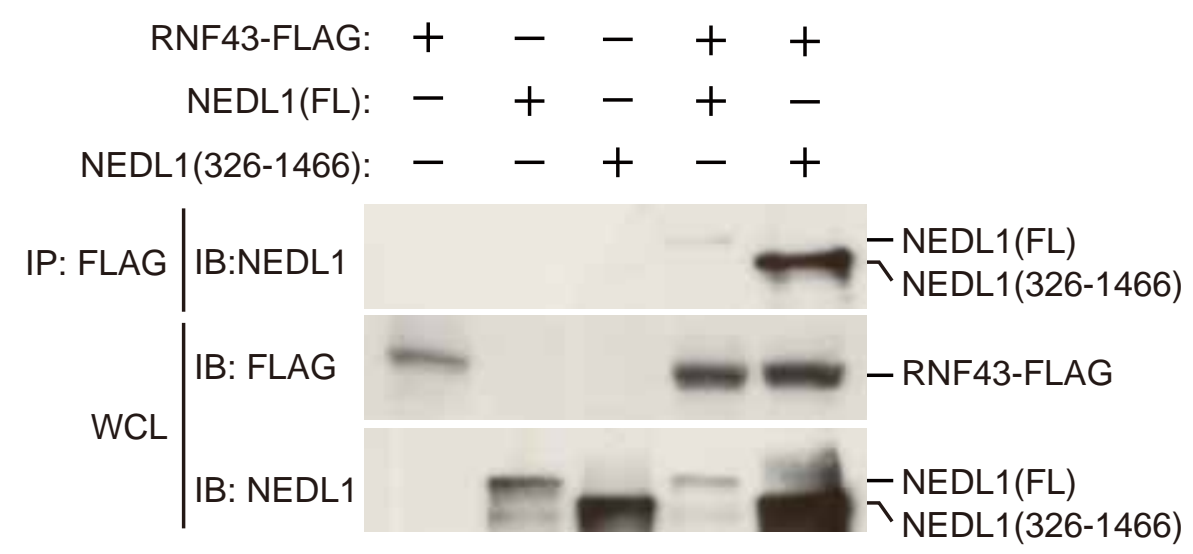

Shinada et al. Figure 1 
A

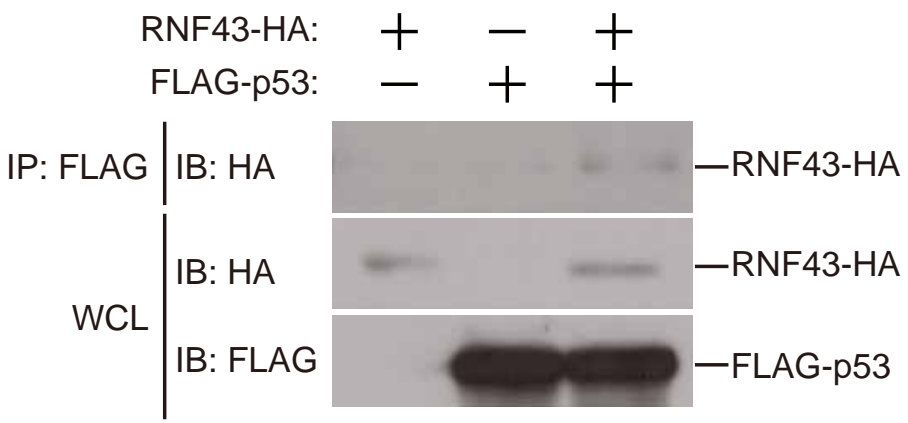

B

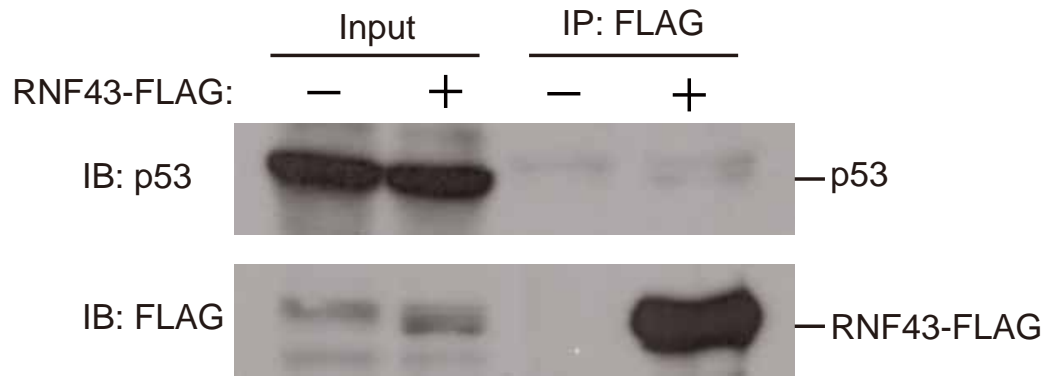

Shinada et al. Figure 2 


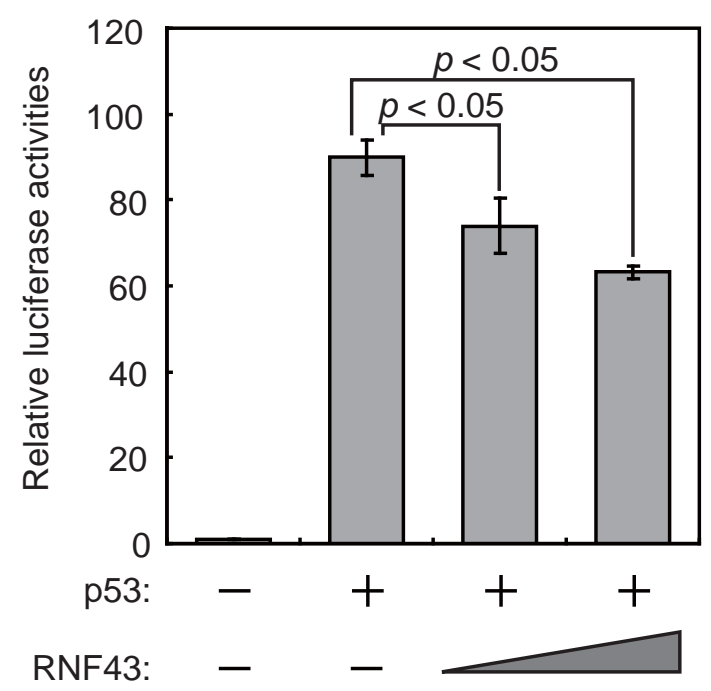

Shinada et al. Figure 3 
A

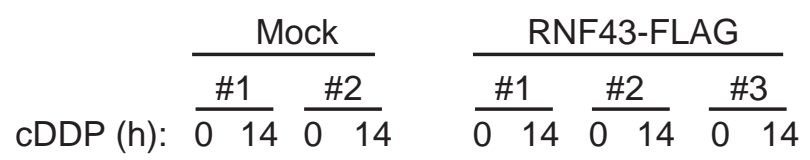

IB: FLAG

IB: p53

IB: Caspase-3

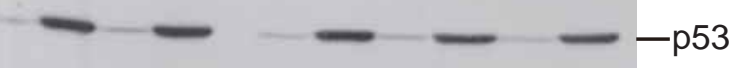

IB: Cleaved caspase-3
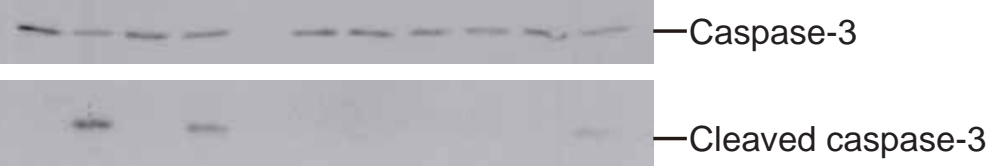

IB: $\beta$-actin

\section{$-\beta$-actin}

B

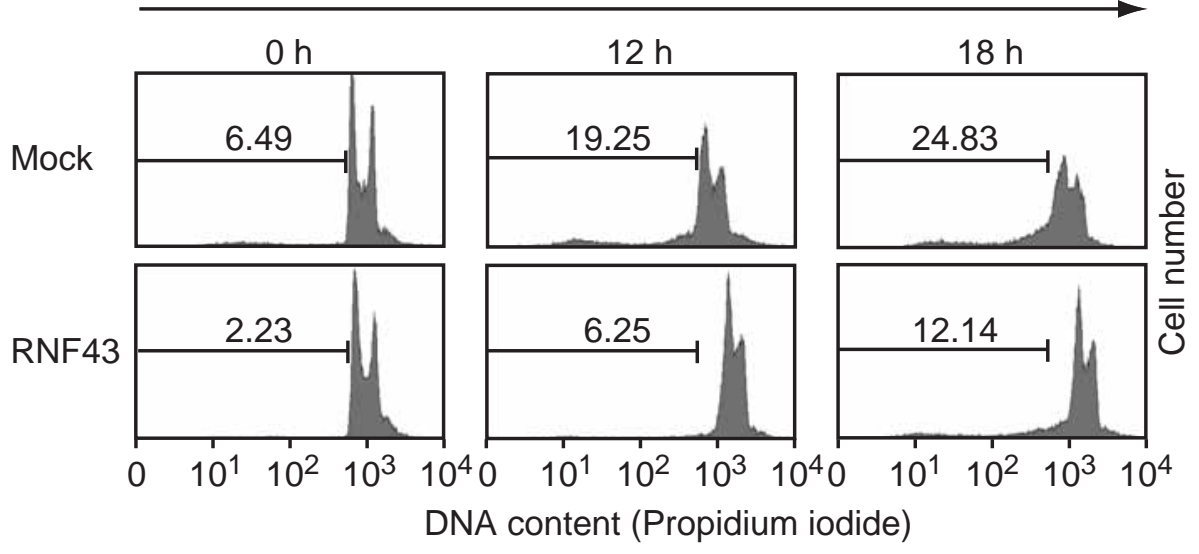

Shinada et al. Figure 4 